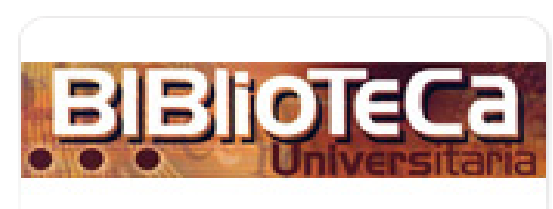

Biblioteca Universitaria

ISSN: 0187-750X

public@dgb.unam.mx

Universidad Nacional Autónoma de México

México

García Martínez, Bardo Javier

Revista Biblioteca Universitaria electrónica: iniciativa Open Journal Systems

Biblioteca Universitaria, vol. 14, núm. 2, julio-diciembre, 2011, pp. 145-155

Universidad Nacional Autónoma de México

Distrito Federal, México

Disponible en: http://www.redalyc.org/articulo.oa?id=28521613003

- Cómo citar el artículo

- Número completo

- Más información del artículo

Página de la revista en redalyc.org

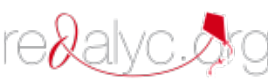

Sistema de Información Científica

Red de Revistas Científicas de América Latina, el Caribe, España y Portugal

Proyecto académico sin fines de lucro, desarrollado bajo la iniciativa de acceso abierto 


\section{Revista Biblioteca Universitaria electrónica: iniciativa Open Journal Systems}

The electronic journal "Biblioteca Universitaria":
Open Journal Systems initiative.

\section{Bardo Javier García Martínez*}

\section{ReSUInER}

Actualmente la Secretaría General de la Universidad Nacional Autónoma de México (UNAM) lleva a cabo una iniciativa para integrar y administrar las revistas académicas electrónicas de la UNAM mediante el gestor de contenidos Open Journal Systems, un sistema especializado en revistas electrónicas; durante la xxxiI Feria internacional del Libro del Palacio de Minería se le dio un gran impulso a este proyecto al presentar el Portal de Revistas Científicas y Arbitradas de la UNAM, el cual pretende alojar, apoyar y dar una amplia visibilidad a las revistas publicadas en dicho portal.

El presente artículo tiene como objetivo dar a conocer los esfuerzos de la Dirección General de Bibliotecas por integrar su revista Biblioteca Universitaria en su versión electrónica al Portal de Revistas Científicas y Arbitradas de la UNAM, y con ello formar parte de las tendencias actuales y cumplir con los requerimientos y estándares de las revistas electrónicas académicas internacionales. De esta manera, pretendemos darle una mayor visibilidad en las bases de datos suscritas, así como ofrecer a nuestros lectores herramientas especializadas para su lectura.

Palabras Clave: Open Journal Systems, ojs, gestor de contenidos, CMS, revistas electrónicas, Revistas Científicas y Arbitradas de la UNAM, Biblioteca Universitaria

\section{Abstract}

Currently, the General Secretariat of the National University of Mexico carries out an initiative to integrate and manage its electronic journals by means of the content management system: "Open Journal Systems", which specializes in electronic journals; during the 32nd International Book Fair of the "Palacio de Minería”, this project was given a big impetus when the Portal of Scientific and Peer-reviewed unam Journals was presented, which seeks to host, support and give a wide visibility to the journals published in the portal

This article aims to raise awareness of the efforts of the General Directorate of Libraries to integrate its journal "Biblioteca Universitaria", in its electronic version, in the Portal of Scientific and Peer-reviewed UNAM Journals, and thus become part of the current trends in publishing and meet the requirements and standards of international academic electronic journals. In this way we aim to give it a greater visibility in the subscribed databases by UNAM as well as providing our readers with specialized tools for reading.

KEYwords: Open Journal Systems, ojs, Content Management Systems, cms, electronic journals, Scientific and Peer-reviewed unAm Journals, University Library

Departamento de Publicaciones de la Dirección General de Bibliotecas, unam. Noveno piso del Edificio de Biblioteca Central, Circuito Interior, C.U., C.P. 04510, México, D.F., México. Correo electrónico: bardo@dgb.unam.mx. 


\section{Introducción}

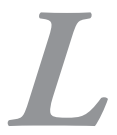

a Dirección General de Bibliotecas es la dependencia universitaria encargada de coordinar a todo el Sistema Bibliotecario y de Información de la unam (SIBIUNAM). Ofrece a la comunidad universitaria de la UNAM y a sus usuarios externos una serie de servicios enfocados a atender y resolver sus necesidades de información.

Uno de sus servicios es la edición y publicación de la presente revista, Biblioteca Universitaria, revista académica técnico-profesional que se publica de forma impresa con una periodicidad semestral. Posterior a su publicación impresa esta misma revista se presenta en su versión electrónica.

Biblioteca Universitaria, al tratarse de una revista académica precisa cumplir con las normas y estándares actuales presentes en la publicación de revistas electrónicas, de la misma forma que su versión impresa, ya que deben complementarse una con la otra.

Actualmente la Secretaría General de la UNAM coordina un proyecto en el cual se lleva a cabo en forma gradual la migración de todas las revistas electrónicas de la UNAM al sistema Open Journal Systems, impulsando con ello el uso estandarizado de gestores de contenido como una herramienta práctica y especializada.

Este objetivo nos ha obligado a replantearnos el modelo anterior de publicación electrónica de la revista, no sólo en cuestión de diseño sino también en cuestión del flujo de trabajo para llevarla a cabo $y$, aún más importante, el formato en que se ofrece el contenido al público.

Con base en ello, Biblioteca Universitaria inicia su inclusión en el sistema ojs como parte del portal de revistas científicas y arbitradas de la UNAM y se puede consultar en la siguiente dirección electrónica:

http://revistas.unam.mx/index.php/rbu

\section{Revista Biblioteca Universitaria versión electrónica}

La revista Biblioteca Universitaria, que como se ha mencionado es una revista de carácter académico, está enfocada principalmente a estudiantes, docentes, egresados y personal académico del SIBIUNAM; sin embargo, este enfoque no está cerrado ya que cuenta con colaboraciones de otras instituciones. Su objetivo y la temática y estructura de sus artículos enfocados a fortalecer la comunicación dentro del SIBIUNAM, la coloca en el rubro de revista académica técnico-profesional.

El propósito de la versión electrónica de Biblioteca Universitaria es ampliar su difusión, ofrecer a los usuarios resúmenes en español e inglés, contar con información acerca de la publicación, proporcionar instrucciones a los autores que deseen enviar su material para publicación, proveer tanto herramientas de lectura como referencias bibliográficas, indicar maneras de citar artículos publicados en ella, ofrecer información bibliográfica de los autores, dar acceso a los textos completos en formato PDF, e integrar en su sitio algunas herramientas de búsqueda y recuperación avanzadas a fin de facilitar la navegación y obtención de sus documentos.

\section{Open Journal Systems (ous)}

El sistema utilizado para alojar y publicar la revista electrónica en esta nueva etapa es un gestor de contenido (en inglés Content Management System, abreviado $\mathrm{CMS}$ ); este sistema podemos describirlo de la siguiente forma:

"Content Management o CM, puede ser definido simplemente como un proceso de recopilar, organizar, categorizar y estructurar fuentes de información de cualquier tipo y formato para que puedan ser almacenadas, extraídas, publicadas, actualizadas y reutilizadas de cualquier forma deseada."1

1 Traducido de: Yu, Holly. Content and Workflow Management for Library Web Sites. Information Science. Los Angeles, USA: California State University, 2005, p. 2. 


\section{ats ocs ous ois \\ PKP | PUBLIC KNOWLEDGe PROJECT} Ner wai rean

\begin{tabular}{|l|}
\hline Search this site: \\
\hline Search \\
\hline
\end{tabular}

Los Sistemas de Gestión de Contenidos consisten en una aplicación electrónica que permite crear una estructura de trabajo para la creación, publicación y administración de contenidos, sus características más atractivas son su facilidad de uso y la capacidad de cooperación grupal para su administración.

Esto funciona como una instalación en un servidor que se comunica con el administrador o editor mediante una interfaz web, por lo general sencilla y de fácil manejo. El sistema permite manejar de manera independiente el contenido y el diseño, lo que facilita agregar información al contenido y modificar el diseño en cualquier momento sin afectar uno al otro, esto es debido a que esa interfaz controla y almacena directamente la información en una base de datos.

Esta clase de sistemas se basan en un principio de cooperación entre múltiples usuarios para su administración; la publicación del contenido es controlada por uno o varios editores, a quienes se les proporciona las herramientas necesarias para ello, permitiendo distribuir el flujo de trabajo.
Open Journal Systems es un gestor especializado en revistas electrónicas, fue desarrollado por el Public Knowledge Project (PKP) y liberado en el año 2002; mantiene la idea de impulsar la nueva generación de publicaciones electrónicas con el criterio comunitario de libre acceso (Open Access), este principio se basa en la idea de publicar electrónicamente contenido académico confiable y sin restricciones de acceso.

El software utilizado en el oss es de código abierto, lo que significa que los usuarios del mismo son libres de modificarlo, por lo cual PKP invita a todos los usuarios del sistema a compartir sus cambios y desarrollos del código con el resto de la comunidad, para ello ofrece en su sitio web foros de discusión y secciones de soporte técnico.

Este sistema permite a los gestores configurar personalmente los requerimientos del flujo de trabajo, secciones y proceso de revisión para la publicación de las revistas alojadas, permite gestionar el envío de artículos de los autores para revisión, corrección y su posterior autorización. 
Su ventaja fundamental es la reducción de tiempo y esfuerzo que generalmente se dedica al proceso editorial de una revista electrónica trabajada de manera convencional. Esta herramienta ya lleva tiempo disponible para cualquier revista del mundo, y cada vez es mucho mayor el número de revistas electrónicas que utilizan este sistema.

Entre las características relevantes para la administración del proceso editorial de la revista y su consulta, oJs ofrece lo siguiente:

- Mantiene una secuencia lógica y controlada durante el proceso de trabajo editorial.

- Permite asignar roles y autorización para el desarrollo de funciones especificas a las personas que intervienen en la edición de la revista.

- Es un sistema flexible para la publicación de revistas previamente editadas y publicadas en otras plataformas, lo que facilita y optimiza los tiempos requeridos para la migración de esos contenidos.

- Desde el punto de vista del usuario mantiene un orden comprensible y de fácil navegación al mostrar los contenidos de una forma coherente.

- Integra una funcionalidad de búsqueda para facilitar la localización de contenidos de interés de los lectores y, por lo tanto, facilita el acceso a la información contenida en la publicación.

\section{Contenido de Biblioteca Universitaria electrónica}

La nueva presentación de Biblioteca Universitaria electrónica incluye el acceso en texto completo de los artículos disponibles en PDF e información detallada de los volúmenes publicados, de los autores que la respaldan

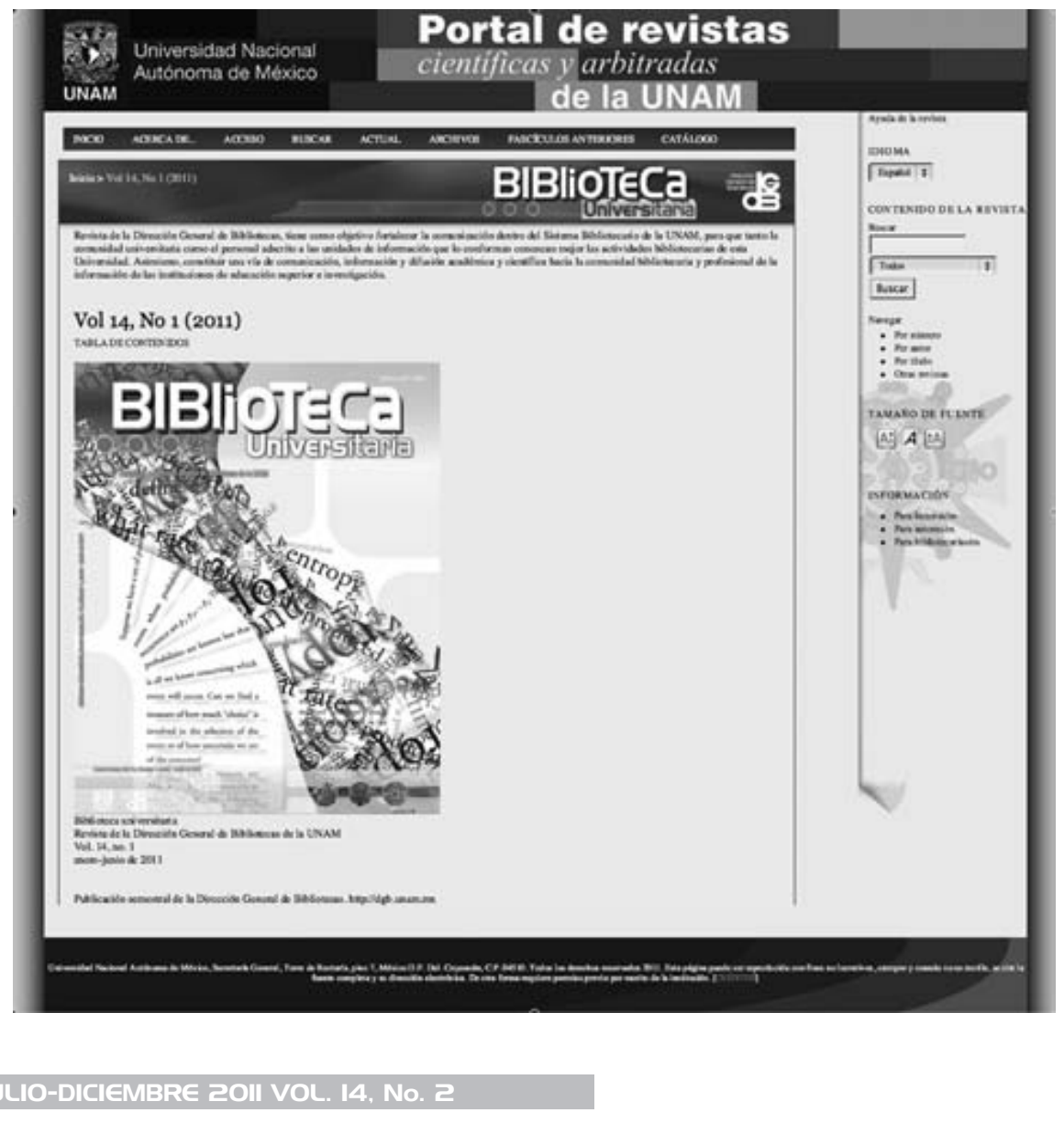


y la dependencia que edita y publica la revista. Ofrece también al usuario una serie de herramientas de lectura que complementan la experiencia de visita al sitio de la revista, así como información de meta-etiquetas dentro del sitio y para cada artículo publicado, para con ello incrementar su visibilidad en los buscadores y bases de datos en las que se encuentra indexada.

Las secciones disponibles para navegación del sitio de la revista son las siguientes:

1. Acerca de la revista: Dentro de este rubro podemos encontrar la información correspondiente a:

- Personas: contacto y equipo editorial.

- Políticas: temática y alcance, así como las políticas de secciones.

- Envíos: envíos online, normas para autores/as y las notas de copyright.

- Otro: mapa del sitio y acerca de este sistema de publicación (oss).

2. Acceso: sección que permite ingresar al sitio como usuario ya registrado.

3. Buscar: herramienta para búsquedas avanzadas dentro del sitio.

4. Actual: presenta el Número en curso de la revista.

5. Archivos: índice de todos los números publicados en el sitio.

Al visitar los artículos de la revista electrónica los lectores pueden encontrar:

- Resumen y abstract.

- Texto completo en formato PDF.

\section{Herramientas de lectura}

En la sección de Herramientas de lectura dentro de los artículos, la información se despliega en ventanas emergentes, permitiendo al usuario consultar otros elementos de su interés y volver al documento que estaba consultando sin perder su ubicación previa:

- Imprimir este artículo. Cada artículo, en su resumen y abstract, cuenta con su versión específica para impresión; el artículo en texto completo, al abrirse en formato PDF, permite también su impresión.

- Información de indexación. Muestra todos los metadatos del artículo que sirven para indexarlo en los buscadores a los que se encuentre registrado.

- Información bibliográfica. Presenta la forma de citar el artículo actual, cuenta también con distintos formatos de citación a elegir.

- Buscar referencias. Herramienta que permite realizar búsquedas de referencias sobre el autor o título del artlculo dentro de los buscadores Google Scholar y Windows Live Academic.

- Política de revisión. Lleva al lector a la sección del sitio Temática y alcance, donde se describe a detalle esta información sobre la revista.

Adicionalmente, el sistema puede ofrecer las Herramientas de elementos relacionados, esto activa una serie de opciones que le permiten al lector realizar búsquedas en sitios o bases de datos especializadas sobre algún término específico dentro del artículo de su interés.

Existen también otras alternativas que ofrece el sistema en los casos en que el criterio de la revista así lo permita:

- Acerca del autor. Muestra el perfil profesional de los autor(es) de cualquier artículo publicado.

- Notificar a un colega. Opción que permite recomendar el artículo por correo electrónico a 


\section{IDIOMA}

Español †

HERRAMIENTAS DEL ARTÍCULO

Imprimir este artículo

i. Informacion de indexación

Información bibliográfica

Buscar referencias

Politica de Revisión

\section{CONTENIDO DE LA REVISTA}

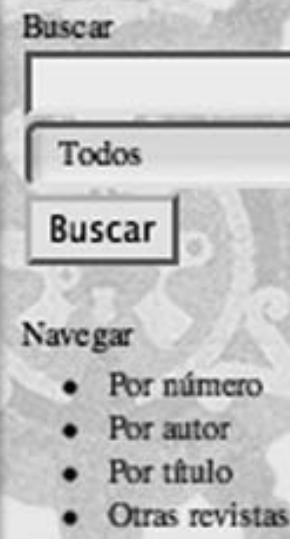

TAMAÑO DE FUENTE

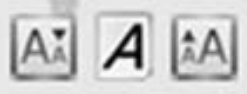

\section{INFORMACION}

- Para lectoras/es

- Para autorasios

- Para bibliotocariasbos

\section{Información bibliográfica}

Las normas mexicanas de catalogación de acervos videográfic y documentos fonográficos, ¿visión a corto o largo plazo?

Formato de cita ABNT :

RODRIGUEZ GARCIA, A., GONZILEZ CASTILLO, R.. Las nomas mexicanas de catalogacín de ac videográficos y documentos fonogríficos, ¿ visión a corto o largo plaxo? Biblioteca Univenitaria,

Norteanérica, $14, \operatorname{sep}$. 2011. Disponible en 〈hitp $/ / \pi v i s t a s$ unam mx/index php/rba/article/view/27 1600 de acceso: 09 Dec. 2011.

\section{Cerrar}

otras personas, es necesario estar registrado en el sitio para acceder a esta opción.

- Mandar correo-e a autor/a. Permite contactar al autor del artículo actual mediante una sencilla forma de envío de datos, también es necesario estar registrado en el sitio para acceder a esta opción.

\section{Proceso editorial}

Los distintos CMS que se encuentran actualmente abiertos al público (tanto comerciales como de código abierto) presentan características similares entre sí, ya sea en el flujo de trabajo, las herramientas administrativas, etcétera. Sin embargo oss, al tratarse de un sistema especializado en su campo, ofrece un flujo de trabajo único en el cual se mueve el envío de artículos de la revista a través de 4 pasos generales que representan el proceso editorial del fascículo, gestionados por uno o más editores:

1. Asignación. El autor proporciona su documento y es asignado al editor.

2. Revisión. Los envíos pasan por una revisión por pares y un proceso de decisión editorial, dentro del sistema se completa o 
modifica la información de meta-etiquetas del artículo según sea conveniente.

3. Edición. Los envíos pasan por corrección de formato, estilo y precisión del texto, no se cambia el sentido o la sustancia del texto.
4. Publicación. Los envíos son asignados a un fascículo y se añade el número de páginas de cada uno.

Como parte de la ayuda al usuario, el propio sistema nos proporciona un esquema para ejemplificar dicho proceso:

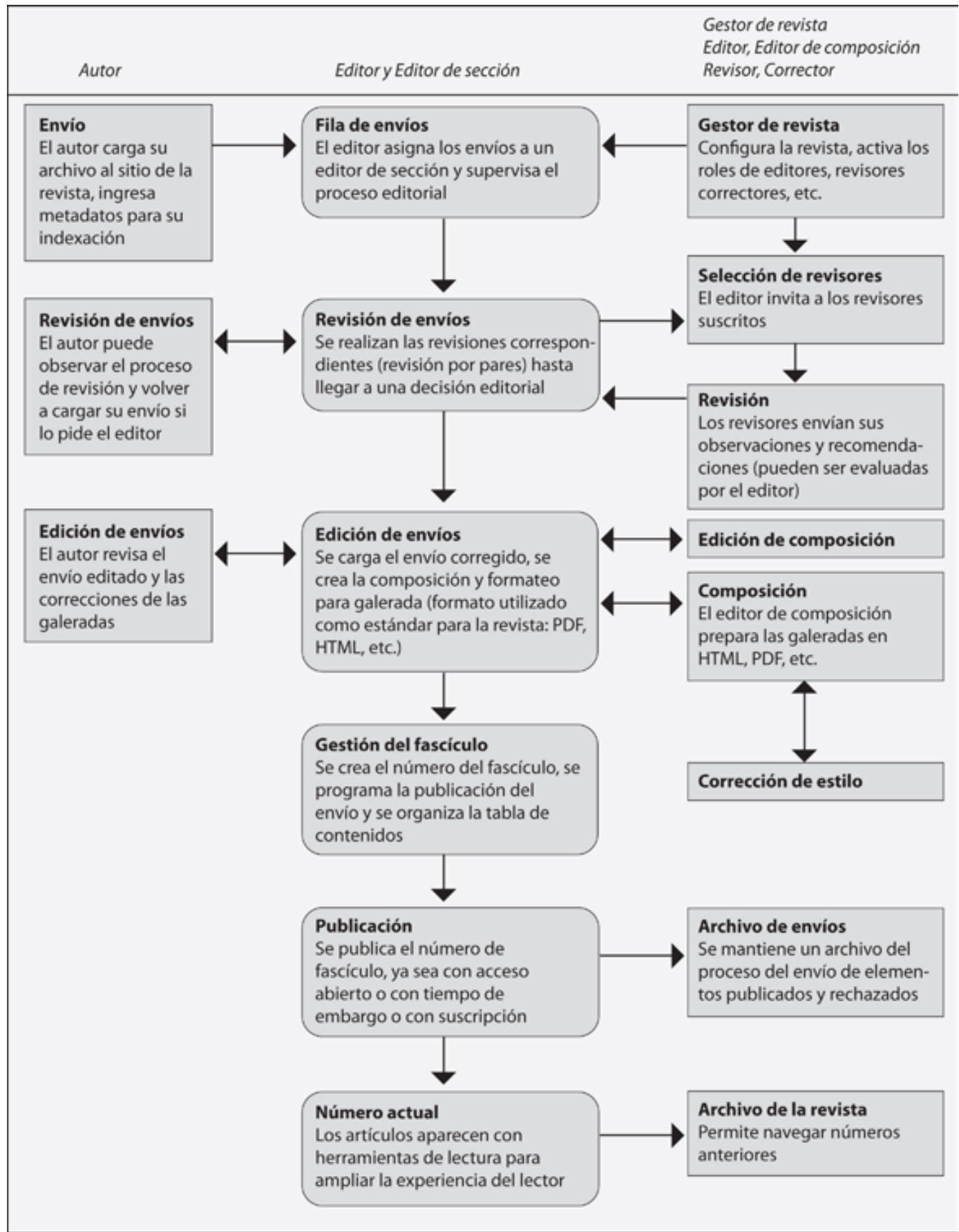




\section{Roles y responsabilidades}

Open Journal Systems constituye una elección acertada como soporte para las revistas electrónicas de la UNAM; al tratarse de un software especializado controla el proceso de trabajo entre los distintos roles que existen como autor, revisor, editor, gestor y administrador; es a la vez un sistema flexible, ya que nos permite la selección y utilización de los roles que se ven involucrados en la publicación de revistas que han sido previamente editadas y publicadas, ya sea en formato impreso o electrónico, lo cual facilita y optimiza los tiempos de trabajo.

Sin embargo, las distintas responsabilidades durante el proceso en oss pueden ser distribuidas entre una o más personas, de acuerdo a las necesidades de la revista; la flexibilidad del sistema nos permite crear, eliminar o fusionar usuarios dentro de los diferentes roles que sean necesarios.

Los roles que se utilizan en oJs son los siguientes:

- Administrador. La persona encargada de crear las revistas del sitio, da soporte técnico a los gestores de la revista y sus privilegios le permiten configurar las características del sitio oss en general.

- Gestor(a) de revistas. Gestiona el sistema de publicación en general, no necesita habilidades técnicas avanzadas, sus actividades consisten en llenar los contenidos del sitio y sus revistas, así como administrar y cargar los archivos pertinentes al sistema. Prepara la revista y asigna a los editores, editores de sección y composición y revisores; establece las políticas y procedimientos de la revista.

- Gestor(a) de suscripción. Se encarga de administrar la suscripción de los autores y lectores a la revista, ya sea por medio de cobro o sólo por registro; este sistema de suscripción también sirve para permitir el envío de artículos.
- Editor(a). Supervisa todo el proceso editorial y de publicación, asigna los envíos al editor de sección y se mantiene al pendiente de la revisión y edición de los envíos. Puede asumir el rol de editor de sección y gestionar los envíos a través de la edición de composición o maquetación, corrección y revisión.

- Editor(a) de sección. Administra las revisiones y la edición de los envíos de las secciones (por ejemplo artículos, reseñas, noticias, etcétera) especificas a las que fue asignado. Si un artículo fue aceptado para publicarse, el editor de sección supervisa el proceso editorial.

- Revisor(a). Es seleccionado por el editor de sección y se encarga de revisar los envíos de los autores, el criterio de la revisión depende de las políticas de la revista.

- Autor(a). Registra y envía sus aportaciones a la revista a través del sistema oss, debe enviar también los metadatos e información de indexación de su artículo a fin de facilitar y mejorar su búsqueda en línea.

- Lector(a). Como su nombre lo indica, permite que el usuario ingrese y lea el contenido del sitio y los fascículos publicados en el sistema, esto en caso de que la revista no sea abierta y requiera suscripción para leer los contenidos.

\section{Indexación y preservación}

La publicación de la revista en este sistema nos ofrece toda una serie de prestaciones en la parte de indexación de la información, para ello se adhiere al protocolo de Recogida de Metadatos de la Iniciativa de Archivos Abiertos. Este protocolo es una norma emergente que sirve para proporcionar acceso a recursos electrónicos de investigación que se encuentran indexados a escala global; esto significa que la persona encargada de la gestión de la revista puede seleccionar especificamente las categorías que desea indexar. 
Si se desea ingresar la revista para indexación de sus contenidos en el sistema de bases de datos de investigación global, se puede registrar la URL de la revista en el Public Knowledge Project metadata harvester. Esta herramienta recopila los metadatos de cada elemento indexado, permitiendo una búsqueda precisa y exhaustiva de los sitios de investigación que se adhieren al Protocolo de Recopilación de Metadatos de la Iniciativa de Archivos Abiertos (Open Access). Los campos de indexación que nos permite utilizar el oss son por disciplina y subdisciplinas académicas, clasificación por materias, palabras clave, cobertura y método enfoque.

oJs también permite indexar la revista con el sistema DoI (Identificador de Objeto Digital) el cual está asignado por CrossRef, una asociación de membresía independiente fundada y dirigida por editores, con la ideología de permitirles trabajar de manera colectiva mediante una red de citaciones vinculadas entre sí de millones de artículos y otros contenidos académicos y de editores profesionales.

Otra opción que nos ofrece Open Journal Systems es su capacidad de soporte para el sistema Lockss (Lots of Copies Keep Stuff Safe), lo que permite asegurar la preservación de la revista de manera permanente, así como la prevención y reparación de posibles daños o corrupciones en los archivos.

"LOCKSS es software libre desarrollado por la Biblioteca de la Universidad de Stanford, que permite a las bibliotecas preservar revistas basadas en web seleccionadas recopilando regularmente los contenidos de las revistas registradas para recopilar el nuevo contenido publicado y archivarlo. Cada archivo es validado continuamente contra el caché de otras bibliotecas, y si el contenido está corrupto o se ha perdido se usan los otros cachés de la revista para recuperarlo."

2 Tomado de la información de sistema en ojs: Portal de revistas científicas y arbitradas de la UNAM [en línea]: <http://revistas. unam.mx/index.php/rbu/> [Consulta: enero de 2010].

\section{Visibilidad}

En el ambiente web se considera visible a un sitio cuando cumple de manera eficiente con los requerimientos técnicos de la indexación en su información, para ello es necesario incluir meta-etiquetas con las palabras clave que den una referencia coherente al contenido; se debe incorporar textos alternos que sean significativos de las imágenes utilizadas en la interfaz, hay que cuidar el manejo correcto de las etiquetas de títulos en las cabeceras de las páginas; de ser posible, contar con vínculos hacia paginas certificadas o de importancia global en la materia, $y$, finalmente, debe ofrecer un mapa del sitio.

Este tipo de estructuración de la información sirve para obtener una mejor posición en los resultados de búsqueda de los motores de consulta como Google, Yahoo, Bing, etcétera; con ello es posible lograr una mayor prioridad del sitio en los resultados iniciales de un listado de búsqueda.

Respecto al atributo de la visibilidad, la ventaja que nos ofrece la especialización del oss en revistas académicas es que se puede asignar la información de indexación a cada uno de los artículos de manera independiente al resto de la información del portal, además de cumplir con el resto de los requerimientos antes mencionados, lo cual abre aún más las posibilidades de ser consultados dentro de las búsquedas realizadas en los diferentes motores y bases de datos académicas.

\section{Accesibilidad}

La UNAM está comprometida a ofrecer igualdad de acceso a la información basada en la web, a fin de que puedan consultarla usuarios con discapacidades. El sitio de Biblioteca Universitaria aquí presentado se ajusta a los principios de accesibilidad de la web.

Durante el diseño y configuración del sitio se evitó el uso de gifs animados, animaciones Flash, Shockwave y tecnologías similares, ya que pueden causar problemas de accesibilidad; de la misma forma, se evitó incluir efectos en JavaScript ya que es posible que algunos usuarios desactiven la visualización de este código en sus navegadores. 


\section{Migración}

La revista Biblioteca Universitaria cuenta con volúmenes publicados de manera electrónica desde su volumen IV, número 1 (1989) hasta el volumen 13, número 1 (2010), por lo que es necesario trasladar estos fascículos a la nueva plataforma de publicación y con ello estandarizar la presentación de los mismos. Como se mencionó anteriormente, el Sistema oss controla y ayuda a manejar cada paso del proceso de dicha migración; esta labor puede ser llevada a cabo por una sola persona o puede distribuirse en distintas personas con diferentes roles de acuerdo a sus funciones. Esto le da al proceso de trabajo una flexibilidad notable.

A la fecha de publicación del presente artículo se han integrado al sistema 13 fascículos de la revista, incluyendo el número en curso, correspondiente al Volumen 14, Número 2. La siguiente etapa de migración consiste en ingresar al sitio los números anteriores de forma descendente hasta actualizar en ojs todos los fascículos de la revista publicados de manera impresa hasta el momento.

\section{Conclusiones}

OJS cuenta con las características necesarias para ser el soporte electrónico adecuado de Biblioteca Universitaria, ya que es un software especializado en revistas y posee la opción de constante crecimiento y desarrollo de acuerdo a las nuevas tendencias y tecnologías gracias al apoyo del equipo de desarrollo de PKP y la comunidad que lo utiliza, teniendo con ello el respaldo de personas con amplia experiencia en el uso del gestor. Ofrece sencillez y rapidez para la actualización del sitio, así como facilidad y flexibilidad en el flujo de trabajo, el cual puede realizarse de manera compartida; permite -además- acceso para la modificación y la personalización parcial de la imagen de la revista.
Algunas de las metas que se pretenden lograr con este proyecto es darle a Biblioteca Universitaria el impulso suficiente para que sea consistente y se mantenga a la vanguardia junto con las revistas electrónicas de nivel internacional, así como aumentar su visibilidad en las listas de resultados de los motores de búsqueda principales e incorporarla a bases de datos internacionales; también se busca facilitar su publicación en versión electrónica a tiempo, cumpliendo a la vez con los estándares actuales necesarios, para con ello permitirle ser calificada como de calidad y excelencia editorial por organismos relacionados con el medio. Así, se espera lograr y mantener un prestigio académico relevante que impulse a los autores potenciales a realizar más contribuciones a su contenido. os 


\section{Obras Consultadas}

Asclepio: revista de historia de la medicina y de la ciencia [en línea]. <http://asclepio.revistas.csic.es/index.php/asclepio> [Consulta: septiembre de 2010].

Content and Workflow Management for Library Web Sites: case studies. [Edited by] Holly Yu. Hershey, Pennsylvania: Information Science, 2005. 259 p.

Garlock, Kristen L., Piontek, Sherry. Designing Web Interfaces to Library Services and Resources. Chicago: American Library Association, 1999. 103 p. ISBN 0838907423.

George, Carole A. User-Centered Library Websites: usability evaluation methods. Oxford: Chandos, 2008. 231 p. ISBN 184334359.

ISSN International Center [en línea]. <http://www.issn.org/> [Consulta: enero de 2011].

Latindex [en línea]: Sistema Regional de Información en Línea para Revistas Científicas de América Latina, el Caribe, España y Portugal. <http://www.latindex.unam.mx/> [Consulta: marzo de 2011].

LOCKSS [en línea]: home. [Lots of Copies Keep Stuff Safe]. <http://lockss.stanford.edu/LOckss/Home/> [Consulta: enero de 2011].

oJs in an Hour [en línea]: an introduction to Open Journal. PKP: Public Knowledge Project Simon Fraser University, Library, 2008. 178 p. <http://pkp.sfu.ca/files/OJSinanHour.pdf> [Consulta: agosto de 2010].

Ordorika Sacristán, Imanol, Lozano Espinosa, Francisco Javier, Rodríguez Gómez, Roberto. Las Revistas de Investigación de la unam [en línea]: un Panorama General. Cuadernos de Trabajo de la Dirección General de Evaluación Institucional, mayo de 2009, no. 4. <http://www.dgei.unam.mx/cuaderno4.pdf> [Consulta: enero de 2010].

PKP [en línea]: Public Knowledge Project. <http://pkp.sfu.ca> [Consulta: agosto de 2010].

Revistas Académicas de la UNAM [en línea]. <http://www.revistas.unam.mx> [Consulta: marzo de 2011].

Reyna Espinosa, Felipe Rafael. Revista Biblioteca Universitaria: una década de evolución. Revista Española de Documentación Cientifica, vol. 32, no. 2, 2009, p. 105-114<http://redc.revistas.csic.es/index.php/redc/article/view/462> [Consulta: marzo de 2011].

SciELO [en línea]: Scientific Electronic Library Online. <http://www.scielo.org> [Consulta: enero de 2010]. 